\title{
Autonomous Clustering and Message Passing Protocol for Energy Efficiency in Wireless Sensor Networks
}

\author{
Jenhui Chen and Jiaxing $\mathrm{Xu}$ \\ Department of Computer Science and Information Engineering, \\ Chang Gung University, Kweishan, Taoyuan, Taiwan 333, R.O.C. \\ Email: jhchen@mail.cgu.edu.tw
}

\begin{abstract}
Wireless sensor networks consist of a large number of nodes with limited battery power and sensing components, which can be used for sensing specified events and gather wanted or interesting information via wireless links. It will enable the reliable monitoring of a variety of environments for both civil and military applications. There is a need of energy-efficient message collection and power management methods to prolong the lifetime of the sensor network. Many methods, such as clustering algorithm, are investigated for power saving reason, however, they only consider reducing the amount of message deliveries by clustering but not the load balance of the clusters to extend the maximum lifetime of the network. Therefore, in this paper, we propose a fully distributed, randomized, and adaptable clustering mechanism named autonomous clustering and message passing (ACMP) protocol for improving energy efficiency in wireless sensor networks. ACMP enables sensor nodes cluster themselves autonomously according to their remaining energy and dynamically choose a corresponding cluster head $(\mathrm{CH})$ to transfer the collected information with a minimum energy consumption route. Sensor nodes judge an appropriate power level to form clusters and use minimum energy to exchange messages. The network topology is changed dynamically depending on the CH's energy. Moreover, by maintaining the remaining energy of each node, the traffic load is distributed to all nodes and thus prolong the network lifetime efficiently. Simulation results show that ACMP can achieve a highly energy saving effect as well as prolong the network lifetime.
\end{abstract}

\section{INTRODUCTION}

A wireless sensor network consists of a lot of inexpensive, lower-power, and tiny sensor nodes, which has a wide range of potential applications including environment monitoring, target tracking, security, medical systems, health care, and robotic exploration, etc [5], [9], [13]. These sensor nodes can self-organize to form a network and can communicate with each other using their wireless interface. These sensor nodes are usually unreliable and inaccurate, but their size and cost enable applications to network hundreds or thousands of these tiny sensor nodes in order to achieve high quality, faulttolerant sensing systems. Each node has one or more sensors, embedded processors and low-power radios, and is normally battery operated.

Because of the energy restriction of sensor nodes it needs an energy-efficient communication protocol for battery power saving so that prolongs the sensor network's lifetime. One major task of these sensor nodes is to gather wanted information and send them back to a coordinator called sink node for analyzing and monitoring specific matters. This action will

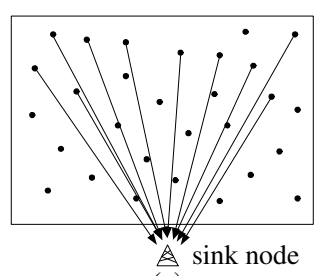

(a)

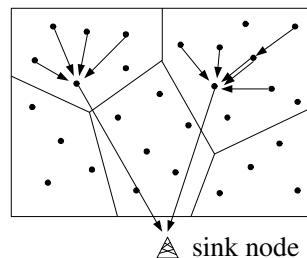

(c)

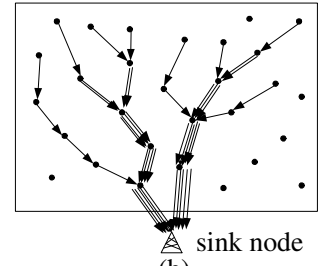

(b)

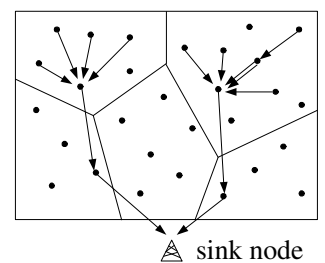

(d)
Fig. 1. An illustration of information gathering with and without clustering: (a) Single hop without clustering; (b) Multihop without clustering; (c) Single hop with clustering; and (d) Multihop with clustering.

consume a lot of energy if there is no efficient communication protocol. One potential solution of saving battery consumption is to reduce the number of messages to the sink node. One simple way to reduce the number of messages is to divide all sensor nodes into several clusters and gathers the information from nodes by cluster head. After accumulating a reasonable amount of messages, cluster heads transfer the aggregated information to the sink node in order to reduce the energy consumption [21]. Fig. 1 depicts an application where sensor nodes periodically transmit information to a remote observer (e.g., a sink node). It shows that the communication overhead can be reduced by separating sensor nodes into several clusters.

Many clustering algorithms have been investigated and proposed in recent years [1], [2], [4], [7], [8], [11], [12], [19]. The Span [4] and geographic adaptive fidelity (GAF) [19] algorithms are geographic topology based clustering protocols that utilize location information to eliminate unnecessary links. However, they may not be feasible since the position of each node is often not provided in practice. The low-energy adaptive clustering hierarchy (LEACH) [11] utilizes randomized rotation of clusterheads (CHs) to evenly distribute the energy load among sensor nodes in the network. In fact, the rotation of $\mathrm{CHs}$ is not necessary and may waste more energy if there are few events in some areas. The clustering-based maximum lifetime 
data aggregation (CMLDA) [8] scheme is a data collection algorithm that focuses on how to find an efficient manner in which the data should be collected from all sensor nodes and transferred to the sink node, such that the network lifetime is maximized. Nevertheless, CMLDA does not considers the total energy usage and thus not achieve the global solutions. The Max-Min $D$-Cluster algorithm [1] generates $d$-hop clusters with a run-time of $O(d)$ rounds. Unfortunately, this algorithm does not ensure that the energy used in communicating information to the sink node is minimized.

In [2] and [7], the authors propose a distributed algorithm for organizing sensors into a hierarchy of clusters with the objective of minimizing the total energy spent in the system to communicate the information gathered by these sensors to the sink node. However, they do not consider the network lifetime, which is defined as the time from nodes deployment to the time when the first node is run out of function due to energy depletion. The energy consumption is defined as the total energy consumed by all nodes in the sensor network during the whole data processing procedure. In [12], the authors propose a dynamic cluster-based structure to track the movement of boundaries and facilitate the fusion and dissemination of boundary information in a sensor network. It is suitable for tracking special events like fire but is not for tracking one or more individual objects, such as people, animals, and vehicles.

To avoid above mentioned drawbacks, this paper proposes an autonomous clustering and message passing (ACMP) protocol for wireless sensor networks. The ACMP has five unique characteristics:

- ACMP is a fully distributed and autonomous sensor communication protocol.

- Each node can join $C$ clusters at most simultaneously.

- The cluster topology is changed dynamically depending on the remaining energy of $\mathrm{CHs}$.

- The load balance of each cluster is considered in this scheme.

- ACMP supports local re-clustering.

Sensor nodes will dynamically decide to pass the data to a $\mathrm{CH}$ which has more remaining energy. It will dynamic recluster locally, if have no need to re-cluster globally. We will simulate it to demonstrate that the energy consumption is fewer and the network lifetime will longer than other clustering protocols. ACMP is a protocol for self-organized and autonomous distributed sensor systems.

The remainder of this paper is organized as follows. Section II describes the system model of ACMP. Section III discribes the detail of ACMP. In Section IV, shows ACMP effectiveness via simulations and compares it to other clustering techniques. we perform a series of simulations to evaluate the performance of ACMP. Finally, we give some conclusions in Section V.

\section{System Model}

ACMP uses the carrier sense multiple access (CSMA) medium access control (MAC) protocol to form clusters.

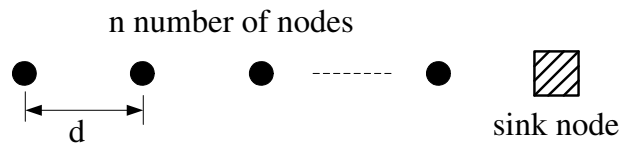

Fig. 2. A simple linear network.

Once the clusters are created, the $\mathrm{CH}$ receives all messages from its members of the cluster. According to the number of its members, the $\mathrm{CH}$ creates a time schedule time based on division multiple access (TDMA) protocol [6], [14], [17] to tell its members when it can wake up to transmit or receive. The radio of each member can be turned off until the node's allocated transmission time, thus it can minimize energy consumption. The $\mathrm{CH}$ must keep its receiver on to receive all the data from the nodes in the cluster. When all the data has been received, the $\mathrm{CH}$ compresses the data into a single message and transmit the aggregated information to the sink node.

A typical sensor node consists mainly of a sensing circuit for signal conditioning and conversion, a digital signal processor, and radio links [3], [10], [15]. The energy consumption model [11], [16] for each sensor are given as below.

\section{A. Communication Energy Dissipation}

The key energy parameters for communication in this model are the energy/bit consumed by the transmitter electronics $\left(\alpha_{t}\right)$, energy dissipated in the transmit op-amp $\left(\alpha_{a}\right)$, and energy/bit consumed by the receiver electronics $\left(\alpha_{r}\right)$. Taking Fig. 2, assume a $d^{2}$ energy loss due to channel transmission. Thus, to transmit a $r$-bit message a distance $d$ using the radio model, the radio expends:

- $E_{T x}(r, d)=\alpha_{t} r+\alpha_{a} r d^{2}$, where $E_{T x}$ is the energy consumed to send a $r$-bit message.

- $E_{R x}(r, d)=\alpha_{r} r$, where $E_{R x}$ is the energy consumed to receive a $r$-bit message.

- $\alpha_{t}, \alpha_{r}$, energy dissipated in transmitter and receiver electronics per bit (Taken to be $50 \mathrm{~nJ} / \mathrm{bit}$ ).

- $\alpha_{a}$, energy dissipated in transmitter amplifier (Taken to be $100 \mathrm{pJ} / \mathrm{bit} / \mathrm{m}^{2}$ ).

- $r$, number of bits in the message.

- $d$, message transmission distance.

\section{B. Computation Energy Dissipation}

We assume the leakage current model of [15], [18]. The model depends on the total capacitance switched and the number of cycles in the program.

\section{Autonomous Clustering And Message Passing}

Consider a sensor network consisting of hundreds or thousands of sensor devices, which are fairly distributed in an area, with the same hardware specification. Each sensor node has $k$ kinds of power level $E=\left\{e_{1}, e_{2}, \ldots, e_{k}\right\}$ and its corresponding transmission distances are $d_{1}, d_{2}, \ldots, d_{k}$. Assume $e_{1}<e_{2}<\ldots<e_{k}$ then we have $d_{1}<d_{2}<\ldots<d_{k}$. Taking Fig. 2, in minimum-transmission-energy (MTE), each 


\begin{tabular}{|l|l|l|l|l|l|l|}
\hline Type & Destination & Source & CH & Remaining Energy & TTL & Hop_Count \\
\hline
\end{tabular}

Fig. 3. The ACMP control message format.

node sends a message to the closest node on the way to the sink node. The node located at distance $n d$ from the sink node would require $n$ transmits a distance $r$ and $n-1$ receives. From the literature [11], it shows that the direct communication to the sink node requires less total energy than MTE routing protocol if:

$$
\frac{\alpha_{t}}{\alpha_{a}}>\frac{d^{2} n}{2} .
$$

According to this criterion, ACMP can choose a minimum energy consumption route to the $\mathrm{CH}$ to form the cluster. In the following, we will describe ACMP in detail.

\section{A. Clustering and Power Control}

Assume each node has the same probability $P$ to become a $\mathrm{CH}$ in the network. Initially, every node decides itself whether to be a $\mathrm{CH}$ or not according to the probability $P$. The node will advertise a control message via broadcast to its neighbors within its radio transmission range with the lowest power level $e_{1}$ once it decides to be a $\mathrm{CH}$. The control message format is shown in Fig. 3 and described as follows:

- The "TYPE" field indicates the type of the message which represents REQUEST, REPLY, REFRESH, or RESET, respectively. The REQUEST message is sent by an unclustered node which wishes to form a cluster; the REPLY message is used to reply to the REQUEST; the REFRESH message is used by $\mathrm{CHs}$ to announce its members to update their $\mathrm{CH}$ table. The RESET message is sent by $\mathrm{CHs}$ to announce its members to exit their cluster and delete the records of $\mathrm{CHs}$ that announce the message.

- The "Destination" is the destination address.

- The "Source" is the sender's address.

- The " $\mathrm{CH}$ " field indicates the message belongs to which $\mathrm{CH}$.

- The "Remaining Energy" is the remaining energy of $\mathrm{CH}$.

An unclustered node wishes to form a cluster, it sends the REQUEST message. Before sending the REQUEST message, the sensor node should proceed a backoff procedure to avoid more than one node serving as a $\mathrm{CH}$ at the same time. We let $B\left(E_{r}\right)$ represent the backoff function and is given by

$$
B\left(E_{r}\right)=\frac{2 m}{E_{r}}
$$

where $m$ is the number of neighbors of the sender and $E_{r}$ is the remaining energy of the node. This strategy is to ensure that the node with a higher remaining energy would serve a $\mathrm{CH}$ first.

Nodes which receive the REQUEST message will become a cluster member automatically and check the TTL field (timeto-life) to determine whether forward this message or not. When the TTL is bigger than 1, it subtracts 1 from TTL and

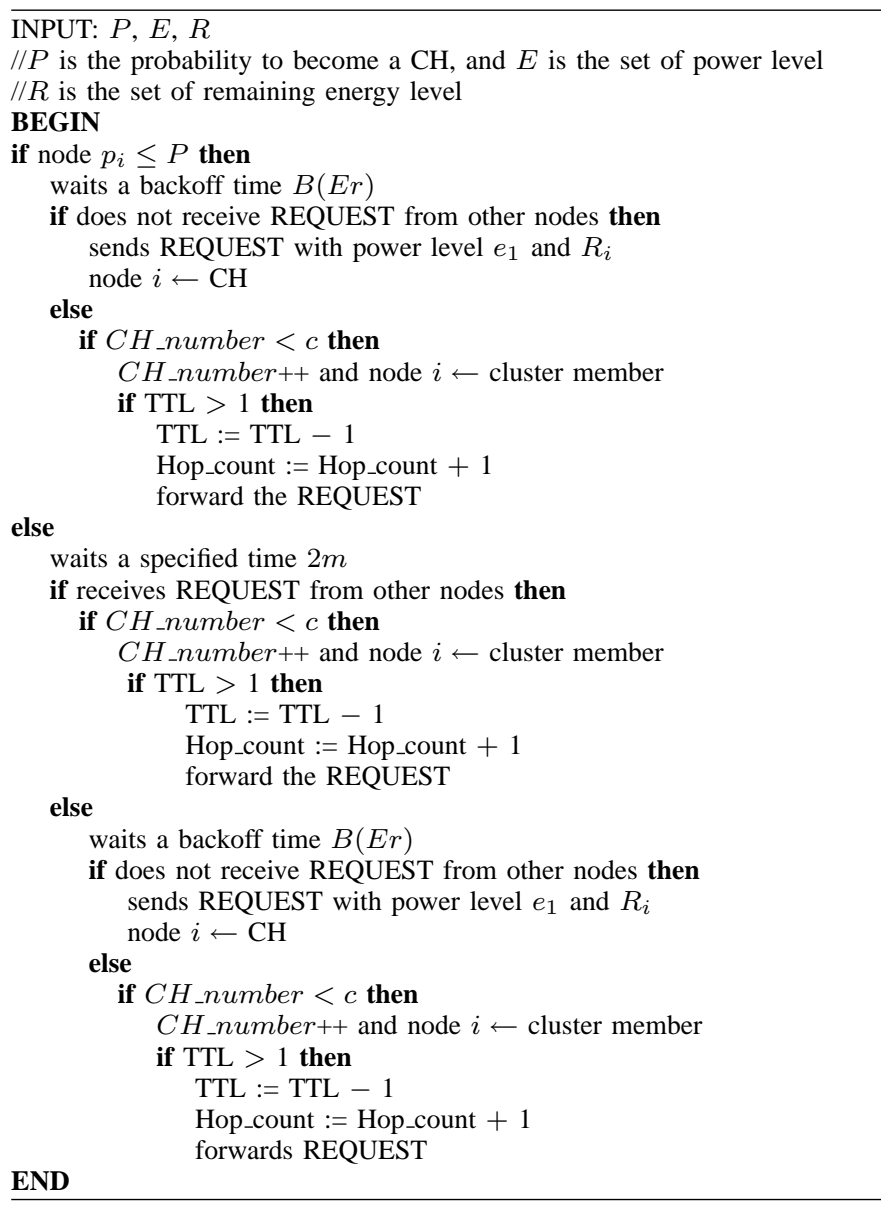

Fig. 4. The clustering algorithm.

forwards this message with minimum transmit energy $e_{1}$ via broadcast to its neighbors. The forwarding process will be terminated until the value of TTL reaches 1 . The Hop_count field will be increased by 1 when forward is performed. This field is provided for sensor node to estimate itself how far from the $\mathrm{CH}$. We note that each node can join $c$ clusters at most simultaneously. A detailed description of the clustering algorithm is shown in Fig. 4.

Fig. 5 illustrates an example of the cluster forming process. Initially, node $\mathrm{A}$ wishes to be a $\mathrm{CH}$ and broadcasts a REQUEST message to its 1-hop neighbors with power level $e_{1}$ and the value of TTL field is 2. If it's 2-hop neighbors do not join any cluster or the number of joined clusters less than $c$, it will join the cluster. At first, nodes $\mathrm{B}, \mathrm{C}$, and $\mathrm{D}$ receive the REQUEST message, and check TTL field. The TTL is 2, nodes B, C, and D subtract 1 from TTL and the Hop_count field be increased by 1 and estimate an appropriate power level to send REPLAY message to the $\mathrm{CH}$ according to the Hop_count and the criterion (1).

Nodes B, C, and D forward this message with power level $e_{1}$ via broadcast to their neighbors. Node $\mathrm{E}, \mathrm{F}$, and $\mathrm{G}$ receive the forward REQUEST message, and check TTL field. The TTL is 1, they estimate an appropriate power level to send REPLY message to the $\mathrm{CH}$ and do not forward the message. 


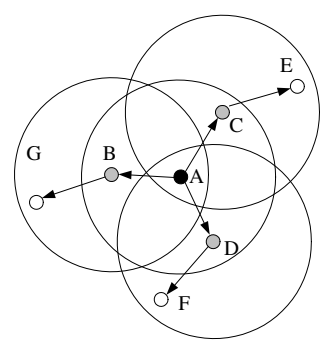

(a)

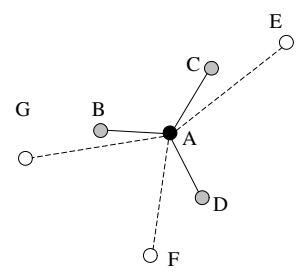

(b)
Fig. 5. The initial clustering, node A uses minimum power level $e_{1}$ to broadcast the REQUEST message to form cluster. The solid node indicates the $\mathrm{CH}$ and the gray and white nodes use power level $e_{1}$ and $e_{2}$ to pass massage, respectively.

In this example, we assume the transmit range of $e_{1}$ and $e_{2}$ are $d_{1}=10 \mathrm{~m}$ and $d_{2}=20 \mathrm{~m}$, respectively. After forming the cluster, nodes $\mathrm{B}, \mathrm{C}$, and $\mathrm{D}$ use $e_{1}$ to pass message to the $\mathrm{CH}$ and nodes $\mathrm{E}, \mathrm{F}$, and $\mathrm{G}$ use $e_{2}$ to pass message to the $\mathrm{CH}$. The result of clustering as shown in Fig. 5(b).

Every node should maintain a $\mathrm{CH}$ table, which records the joined CH's address, the transmit power level to the $\mathrm{CH}$, and the remaining energy of the $\mathrm{CH}$. Each $\mathrm{CH}$ maintains a participation table records the information of the participating nodes in the cluster and the transmit power level to its members.

\section{B. The analysis model}

Assume all sensor nodes are distributed uniformly in a $L \mathrm{~m} \times L \mathrm{~m}$ square area and the diameter of the cluster is represented as $h$-hop. The total energy consumption of the sensor network is the energy consumed by all member nodes sending data to their $\mathrm{CHs}$ and all $\mathrm{CHs}$ sending aggregate data to the sink node. Let $N, N_{C}$, and $N_{M}$ represent the total number of sensor nodes, $\mathrm{CHs}$, and members in a sensor network, respectively, and $N=N_{C}+N_{M}$. The $N_{M}(i)$ is denoted as the number of members within the $i$-hop distance from CHs.

Let $\Delta_{1}$ be the total energy spent by all sensors communicating $r$ bits of data to their respective CHs. Denote $N_{M}^{i j}$ being the number of members, which is $i$ hops distance from its corresponding $\mathrm{CH}$, belong to the $\mathrm{CH} j$. Thus, the total energy spent by all sensors is given by

$$
\Delta_{1}=\sum_{i=1}^{h} \sum_{j=1}^{N_{C}} N_{M}^{i j}\left(E_{T x}\left(r, d_{i}\right)+E_{R x}\left(r, d_{i}\right)\right),
$$

where $d_{i}$ represents the distance of $i$ hops to the $\mathrm{CH}$. Since sensor nodes are distributed uniformly in the $L \mathrm{~m} \times L \mathrm{~m}$ square area and the sink node is placed in the center of the area, then the average distance $\bar{D}$ from CHs to sink node will be

$$
\begin{aligned}
\bar{D} & =\sum_{x=0}^{L} \sum_{y=0}^{L} P_{x y} D_{x y} \\
& =\sum_{x=0}^{L} \sum_{y=0}^{L} \frac{1}{(L+1)^{2}} \sqrt{\left(\frac{L}{2}-x\right)^{2}+\left(\frac{L}{2}-y\right)^{2}},
\end{aligned}
$$

where $P_{x y}$ is the probability of $\mathrm{CHs}$ distributed in the location $(x, y)$, and $D_{x y}$ is the distance from CHs at $(x, y)$ to the sink node.

Let $\Delta_{2}$ be the total energy spent by all $\mathrm{CHs}$ communicating $r$ bits of data to the sink node. From (1), the energy consumption can benefit from direct transmission than multihop transmission if the transmission distance $d$ satisfies

$$
d<\sqrt{\frac{2 \alpha_{t}}{n \alpha_{a}}} .
$$

Since each transmission includes the energy consumptions of both the transmitter and the receiver, thus the more intermediate nodes is involved in forwarding the more energy is consumed. Therefore, the minimum energy consumption is achieved when $n=2$ in a given distance. Thus the maximum transmission distance $d_{\max }$ of each $\mathrm{CH}$ that will benefit from using direct communication than MTE routing protocol if and only if $d_{\max } \leqslant n d$. From (5), we have

$$
d_{\max } \leqslant \sqrt{\frac{2 n \alpha_{t}}{\alpha_{a}}}
$$

For example, in the energy consumption model [11], [16], if $n=2, \alpha_{t}=50 \mathrm{~nJ} / \mathrm{bit}$, and $\alpha_{a}=100 \mathrm{pJ} / \mathrm{bit} / \mathrm{m}^{2}$, the $d_{\max } \approx$ $44.72 \mathrm{~m}$

In the worst-case, $\mathrm{CHs}$ use $d_{\max }$ transmission range to transmit their aggregate data to sink by one hop or multiple hops. Thus, the average number of hops $\bar{h}_{s}$ from $\mathrm{CHs}$ to the sink node is equal to $\bar{D} / d_{\max }$. The total energy spent by all CHs communicating $r$ bits of data to the sink node can be obtained by

$$
\Delta_{2}=N_{C}\left[E_{T x}\left(r, d_{\max }\right) \bar{h}_{s}+E_{R x}\left(r, d_{\max }\right)\left(\bar{h}_{s}-1\right)\right] .
$$

Assume each CH's cover area can be divided into $h$ concentric circles and the width of each section is $d_{1}$. Then the area of the $i$-th concentric circle $A_{i}$ can be calculated as $\left(d_{i}\right)^{2} \pi$. Thus the area of the $i$-th section denoted as $S_{i}$ is given by

$$
\begin{aligned}
S_{i} & =A_{i}-A_{i-1} \\
& =d_{i}^{2} \pi-d_{i-1}^{2} \pi \\
& =i^{2} \pi d_{1}^{2}-(i-1)^{2} \pi d_{1}^{2} \\
& =(2 i-1) \pi d_{1}^{2} .
\end{aligned}
$$

For example, the area of $S_{2}=(2 \times 2-1) \pi d_{1}^{2}=3 \pi d_{1}^{2}$ and $S_{3}=5 \pi d_{1}^{2}$. Thus, the ratio of the number of nodes in the $i$-th section to the overall number of nodes in the $h$-hop cluster is equal to $S_{i} / \pi\left(h d_{1}\right)^{2}=(2 i-1) / h^{2}$. From (3) and (7), the total energy consumption of the overall network will be

$$
\begin{aligned}
\Delta_{1}+\Delta_{2}= & \sum_{i=1}^{h} \sum_{j=1}^{N_{C}} N_{M}^{i j}\left(E_{T x}\left(r, d_{i}\right)+E_{R x}\left(r, d_{i}\right)\right) \\
& +N_{C}\left[E_{T x}\left(r, d_{\max }\right) \bar{h}_{s}+E_{R x}\left(r, d_{\max }\right)\left(\bar{h}_{s}-1\right)\right] \\
= & \sum_{i=1}^{h}\left[\left(N-N_{C}\right)\left(\frac{2 i-1}{h^{2}}\right)\left(E_{T x}\left(r, d_{i}\right)+E_{R x}\left(r, d_{i}\right)\right)\right] \\
& +N_{C}\left[E_{T x}\left(r, d_{\max }\right) \bar{h}_{s}+E_{R x}\left(r, d_{\max }\right)\left(\bar{h}_{s}-1\right)\right] .
\end{aligned}
$$




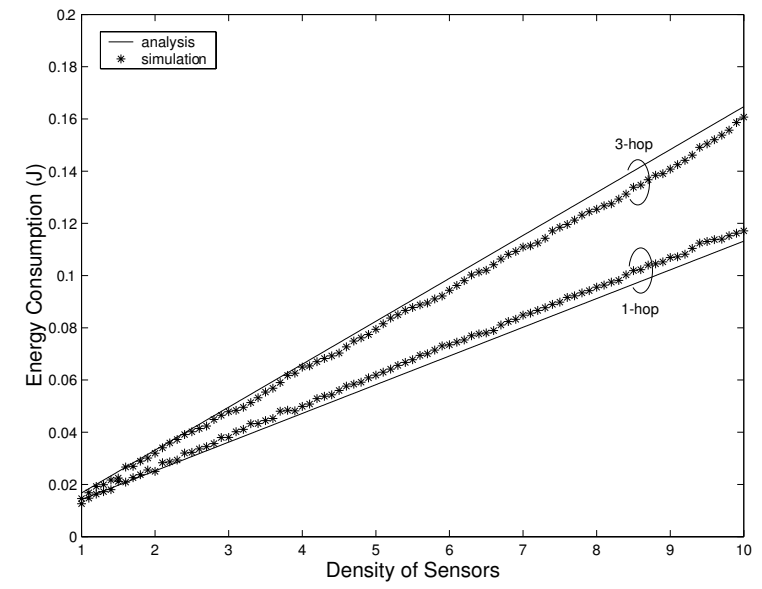

Fig. 6. The results of analysis and simulation of ACMP when $h=1$ and $h=3$.

Now we have to solve the value of $N_{C}$. Assume the radius of a cluster is $h$-hop and denoted as $d_{h}\left(d_{h}=h d_{1}\right)$, the minimum number of $\mathrm{CHs} N_{C \text {,min }}$ that can cover a $\mathrm{Lm} \times L \mathrm{~m}$ square area can be calculated by

$$
N_{C, \min }=\frac{L^{2}}{\pi d_{h}{ }^{2}}=\frac{L^{2}}{\pi h d_{1}{ }^{2}} .
$$

Fig. 6 shows the results of analysis and simulation of ACMP in detail. All nodes are distributed uniformly in $100 \mathrm{~m} \times 100 \mathrm{~m}$ when $h=1$ and $h=3$. We vary the density of sensors from 1 to 10 (100 to 1000 nodes) to investigate the energy consumptions in the result of (9) and ACMP. Assume every node transmit 1 bit of data to their $\mathrm{CHs}$ and after all $\mathrm{CHs}$ aggregate all of the data from member nodes, they send the data to the sink node. We can see that the simulation results are close to our analysis.

\section{Load Balance of $\mathrm{CHs}$}

Clustering enables the network scalability to large number of sensors, reduce the communication overhead and extends the network life. CHs are responsible for coordination among the nodes within their clusters and collection of data information (inter-cluster communication) and sent these data to the sink node. However, the $\mathrm{CH}$ energy will run out quickly if many events occur frequently in its dominated area or it has to coordinate many members in its cluster. We, therefore, propose a load distribution mechanism for load balance of $\mathrm{CHs}$ in ACMP. Assume each $\mathrm{CH}$ has $j$ thresholds of remaining energy $R=\left\{r_{1}, r_{2}, \ldots, r_{j}\right\}$ and $r_{1}>r_{2}>\ldots>r_{j}$.

When the remaining energy of the $\mathrm{CH}$ is less than one of thresholds, it will select the maximum transmit power level recorded in the participation table to broadcast the REFRESH message to announce all of its members. The member nodes will update their $\mathrm{CH}$ table after they receive the REFRESH message. Each cluster member chooses a $\mathrm{CH}$ with a maximum remaining energy according to the $\mathrm{CH}$ table to report data. If there are more than one candidate $\mathrm{CHs}$, i.e., their remaining

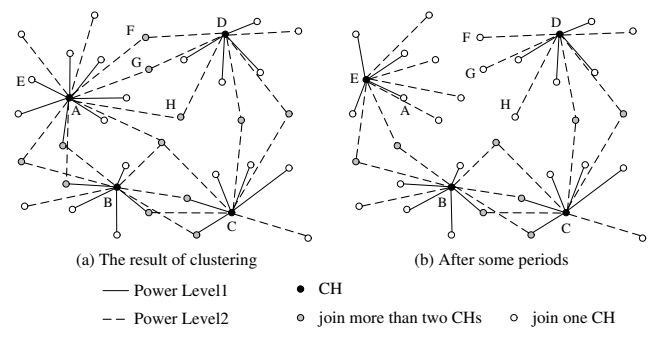

Fig. 7. An example of clustering process of ACMP where cluster size is $20 \mathrm{~m}, h_{1}=10 \mathrm{~m}$ and $h_{2}=20 \mathrm{~m}$. (a) The initial clustering topology. (b) The result of the local re-clustering by node $\mathrm{A}$.

energy are equal, the cluster members choose the closest $\mathrm{CH}$ to report data.

\section{Local Re-clustering}

When the remaining energy of $\mathrm{CHs}$ reaches the lowest energy threshold $r_{j}$, it will choose the maximum transmit power level recorded in the participation table to broadcast the RESET message to all of it cluster members. Each member will join another cluster immediately after receives the message. If nodes do not have any alternative $\mathrm{CH}$ for join and its remaining energy is higher than $r_{j}$, it will form a new cluster with probability $P$. Otherwise, it will serve as a slaver and join its previous $\mathrm{CH}$ again. After local re-clustering, every cluster member will update its $\mathrm{CH}$ table.

\section{E. An Example}

Fig. 7 illustrates an example of clustering process in a sensor network by using ACMP in detail. Initially, all nodes do not join any cluster and each node will form clusters with a given probability $P$ to become a $\mathrm{CH}$ in the network. Assume each node can join 2 clusters at most simultaneously, and the maximum number of hops of each cluster is 2 hops. Fig. 7(a) is the first result of clustering, nodes A, B, C, and D become $\mathrm{CHs}$, each member according the Hop_count to use appropriate power level to communicates with their CHs. After some periods, A's remaining energy lower than $r_{1}$, it broadcasts a RESET message to reset the cluster, and local re-cluster. The result is shown as Fig. 7(b).

\section{Simulation Model and Results}

In order to evaluate the performance of proposed ACMP, a detailed simulation model is developed. First, different numbers of sensor nodes $N$ are uniformly distributed in a $100 \mathrm{~m} \times 100 \mathrm{~m}$ square. These different numbers of sensor nodes 500, 1000, 1500, 2000, 2500, and 3000 are used to represent different network densities $D_{e}$ (see Table I). For example, the $D_{e}=5$ represents the number of sensor nodes is 500 . The size of each cluster is measured by $d_{1}$ (the distance of minimum transmission power) and represented as hops or called TTL. Please notice that, for example, the term "ACMP with 3-hop" implies that the radius of the cluster is 3 hops long and all members within this cluster will use "one hop" to transmit messages to the $\mathrm{CH}$, i.e., direct transmission. Each sensor node can join different number of clusters $C$ simultaneously. 
This implies that a sensor node can join $C$ clusters around its neighboring nodes at most if any.

TABLE I

System PARAmeters in Simulations

\begin{tabular}{lccc}
\hline \hline $\begin{array}{l}\text { Number of } \\
\text { Sensors }(N)\end{array}$ & $\begin{array}{c}\text { Density } \\
\left(D_{e}\right)\end{array}$ & $\begin{array}{c}\text { Probability } \\
(P)\end{array}$ & $\begin{array}{c}\text { Maximum Number } \\
\text { of Hops (TTL) }\end{array}$ \\
\hline 500 & 5 & 0.1012 & 5 \\
1000 & 10 & 0.0792 & 4 \\
1500 & 15 & 0.0688 & 3 \\
2000 & 20 & 0.0622 & 3 \\
2500 & 25 & 0.0576 & 3 \\
3000 & 30 & 0.0541 & 3 \\
\hline \hline
\end{tabular}

The event occurring model in all simulations is generated by a given probability $P_{e}$. The simulated area is divided into many $2 \mathrm{~m} \times 2 \mathrm{~m}$ squares and each of them has the same event occurring probability $P_{e}$. The event is triggered every one round and the round is defined as a specific time unit. Once an event occurs, the sensor nodes around the event will generate messages to transfer to the corresponding CHs. Each sensor node has ten kinds of power levels $e_{1}, e_{2}, \ldots$, and $e_{10}$, and their corresponding transmission distances are $10 \mathrm{~m}, 20 \mathrm{~m}, \ldots$, and $100 \mathrm{~m}$, respectively. The sensing range of each sensor is set $2 \mathrm{~m}$ long and the initial energy of each node is one Joule. There are nine threshold levels of remaining energy $R=\{9 / 10,8 / 10, \ldots, 1 / 10\}$. When the remaining energy of the $\mathrm{CH}$ reaches any threshold of $R$, the $\mathrm{CH}$ will announce this status to its members. Members after receiving this message will choose another $\mathrm{CH}$ for passing collected data if any. The $\mathrm{CH}$ performs the re-clustering procedure locally only when the remaining energy reaches $1 / 10$.

In the simulations, ACMP is compared with the energy efficient hierarchical clustering (EEHC) algorithm [7] and LEACH [11] scheme, to evaluate the performance of power consumption and network lifetime. We refer to the optimal energy minimization parameters of the EEHC algorithm in [7], which is shown in Table I. For comparison, ACMP adopts the same parameters as in EEHC, excepting the parameters $\mathrm{TTL}=3$ and TTL $=1$. The data length of each sensed information is represented as 2000 bits long and reports to the $\mathrm{CH}$ per each event. Each simulation run lasts 50,000 rounds and each simulation result is obtained by averaging the results from ten independent simulation runs.

\section{A. Simulation Results}

In the experiments, we investigate two major metrics as the performance of the protocols:

- Network Lifetime: The time from nodes deployment to the time when the first node is run out of function due to energy depletion. It is measured in rounds.

- Energy Consumption: The total energy consumed by all nodes in the sensor network during the whole data processing procedure.

The first simulation evaluates the network lifetime of the ACMP by varying the parameter $C$. The simulation is terminated immediately when any node runs out of its energy.

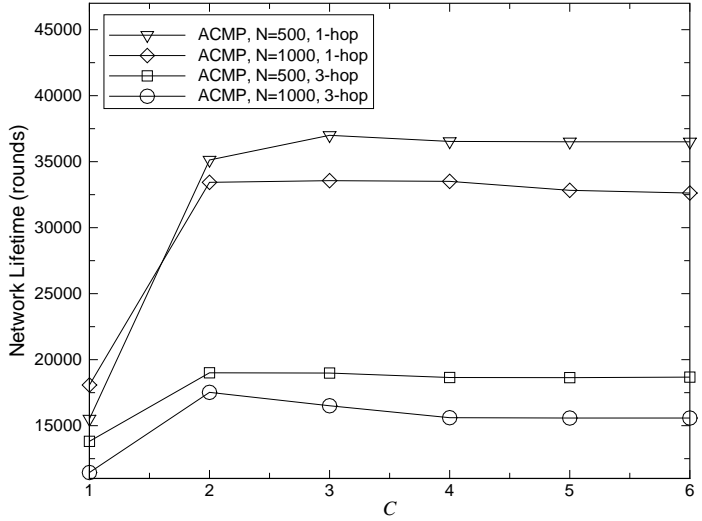

Fig. 8. The network lifetime vs. Max. joinable CHs $(C)$ when $P_{e}=0.1$.

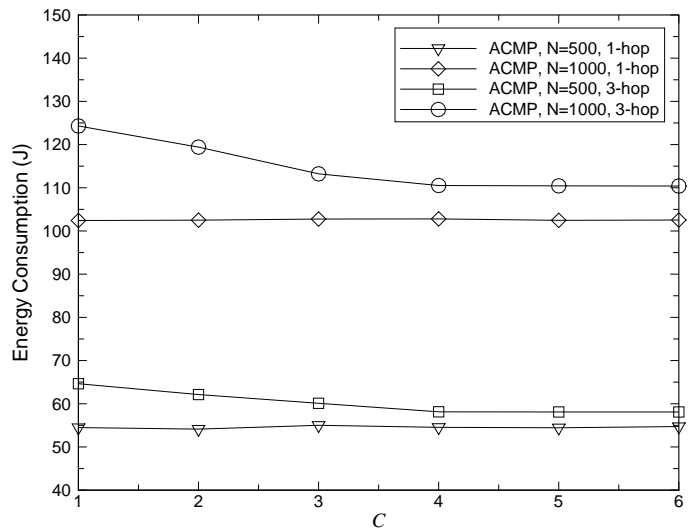

Fig. 9. The energy consumption vs. Max. joinable $\mathrm{CHs}(C)$ when $P_{e}=0.1$ and the simulation time is 15000 rounds.

From the simulation results, shown in Fig. 8, the network lifetime will be longer than that the members can only join one $\mathrm{CH}$. This is because that ACMP can benefit from two main mechanisms: (i) autonomous clustering (dynamic load balance of $\mathrm{CHs}$ ) and (ii) autonomous message passing, and, hence, extends the network lifetime efficiently. The first mechanism enables CHs to re-cluster itself automatically as their remaining energy is lower than each threshold of $R$. This mechanism can prevent the $\mathrm{CH}$ from running out of its energy quickly by taking turns to be the $\mathrm{CH}$ with its neighboring nodes. The second mechanism is a dynamic load balance scheme to alleviate the message forwarding load of CHs. This mechanism is achieved by each sensor node dynamically choosing one of its neighboring $\mathrm{CHs}$, which has the largest remaining energy, to pass the message if the sensor node can join more than one CH simultaneously.

From Fig. 8, we can see that the network lifetime of $C=1$ is lower than that can join more than one $\mathrm{CH}$ both in 1-hop $(\mathrm{TTL}=1)$ and 3-hop cases. ¿From this result, we can know that the network lifetime can be prolonged efficiently when the sensor nodes can choose more than one $\mathrm{CH}$. Moreover, from the results, we can see another interesting remarkable results 
that the network lifetime will be shorter when the cluster size is larger. This is because that the CHs' energy will be run out rapidly due to the cluster size is larger, the $\mathrm{CHs}$ must coordinate more sensors as well as lead to more overheads of the CHs. This strategy will waste more energies and degrade the network lifetime. One the other hand, when the cluster size is smaller, there are fewer sensors in the clusters and the overheads of the $\mathrm{CHs}$ is lower. Thereby if the cluster size is smaller, the network lifetime is longer than the cluster size is larger.

Following above experiment, Fig. 9 shows the energy consumption of the sensor nodes under different $C$. We can see that the energy consumption of $N=1000$ is higher than that of $N=500$. But the energy consumption of 1-hop cases ( $N=500$ and $N=1000$ ) in different $C$ are quite equal. This is because that no matter how the sensor node chooses the $\mathrm{CH}$ for passing messages, the energy consumption is same since the sensor node sends messages to the $\mathrm{CH}$ with power level $e_{1}$. However, in the case of 3-hop, the total energy consumption will decrease when the $C$ increases. Under the case of $C=1$, if the distance between the member and the $\mathrm{CH}$ is far, it has to use higher power level to send messages. This will cost a lot of energy consumptions. On the contrary, when $C>1$, the members can send message to their $\mathrm{CHs}$ according to the remaining energies of $\mathrm{CHs}$ alternately. If the remaining energies of $\mathrm{CHs}$ are equal, the member will randomly choose one $\mathrm{CH}$ to transmit. Because the traffic load is dispersed to each sensor nodes, the network lifetime is prolonged efficiently.

In Fig. 10 and Fig. 11, we vary the $D_{e}$ from 5 to 30 to investigate the energy consumption and network lifetime of ACMP, EEHC, and LEACH. The event occurring probability is set as $P_{e}=0.1$. We observe that, from Fig. 10, EEHC consumes more energies than ACMP 1-hop and 3-hop since ACMP computes a minimum energy consumption route by (1) to report sensed information to the CH. However, EEHC always uses the minimum power to report data to the $\mathrm{CH}$ and involves many intermediate nodes for data forwarding. Meanwhile, LEACH also consumes more energies than ACMP 1-hop and 3-hop since LEACH utilizes randomized rotation of $\mathrm{CHs}$ to evenly distribute the energy consumptions among sensor nodes in the network. Since the rotation of $\mathrm{CHs}$ is periodically performed by EEHC, it will cause more energy consumptions when $P_{e}$ is low. We also note that the gap of energy consumption among ACMP, EEHC, and LEACH gets bigger as $D_{e}$ increases since, in higher density network, more sensor nodes will be involved to forward data and thus consume more energies.

In Fig. 11, the network lifetime of ACMP, EEHC, and LEACH decreases following the increment of the $D_{e}$ (from 5 to 30). This is because that more nodes will sense events and generate the messages to transfer to the sink node in higher density. This outcome lead to shorter network lifetime. However, ACMP uses the load balance to alleviate the traffic overhead of $\mathrm{CHs}$ and expend the lifetime of each node by local re-clustering when its energy is low. Therefore, ACMP

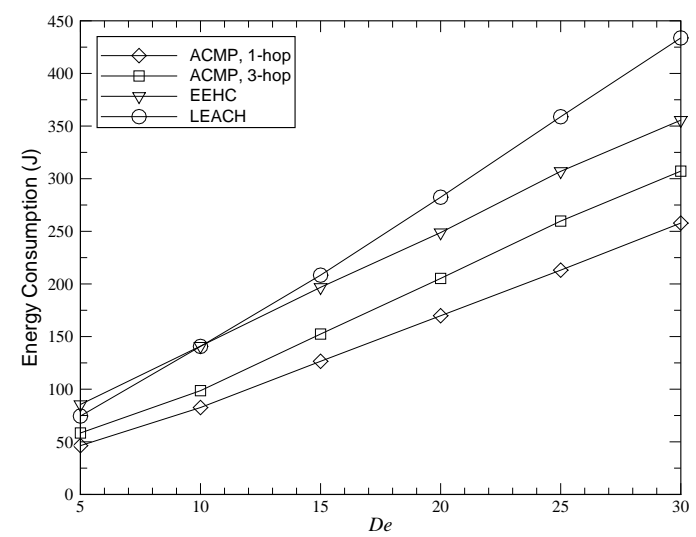

Fig. 10. Density of sensors vs. energy consumption when $P_{e}=0.1$ and the simulation time is 15000 rounds.

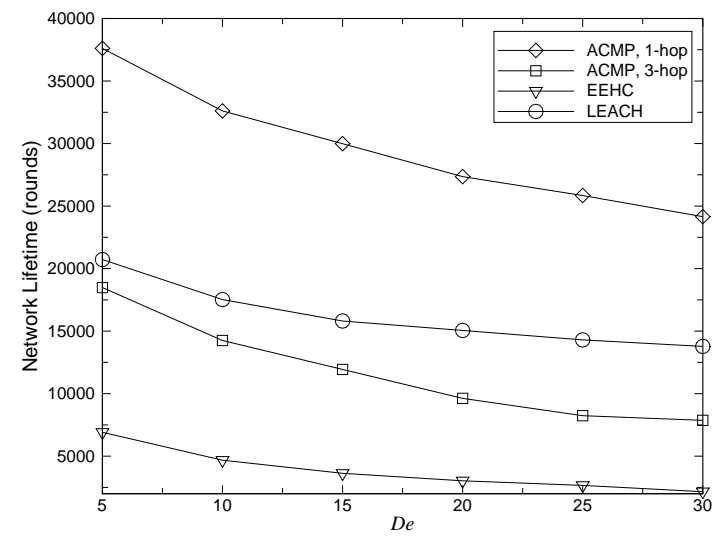

Fig. 11. Density of sensors vs. network lifetime when $P_{e}=0.1$.

can get a longer network lifetime than EEHC. From Fig. 6, we can know ACMP 3-hop spends more energy consumption than ACMP 1-hop, in other words, ACMP 1-hop will have more network lifetime than ACMP 3-hop. The network lifetime of ACMP 1-hop is also longer than LEACH since LEACH utilizes randomly rotation of $\mathrm{CHs}$. This will waste more energies.

To investigate the influence of $P_{e}$ on energy consumption and network lifetime, we perform a detailed experiment by varying $P_{e}$ to observe the results obtained by ACMP, EEHC, and LEACH as shown in Fig. 12 and Fig. 13. We can see that energy consumptions of ACMP, EEHC, and LEACH increase as $P_{e}$ increases. ACMP both in 1-hop and 3-hop can get lower energy consumption than LEACH and EEHC since either LEACH or EEHC will re-cluster themselves periodically. On the contrary, ACMP re-clusters depending on the remaining energy of $\mathrm{CHs}$ and thus saves more energies than LEACH and EEHC. Notice that the gap of energy consumption between ACMP 1-hop and EEHC gets bigger and bigger when $P_{e}$ increases since ACMP utilizes autonomous clustering/reclustering and message passing mechanisms to reduce the probability of one sensor node running out its energy rapidly. 


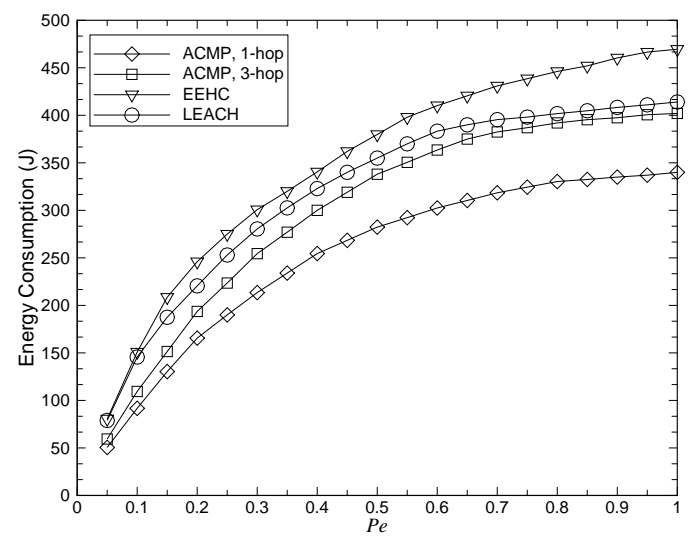

Fig. 12. The $P_{e}$ vs. energy consumption when $N=1000$ and the simulation time is 15000 rounds.

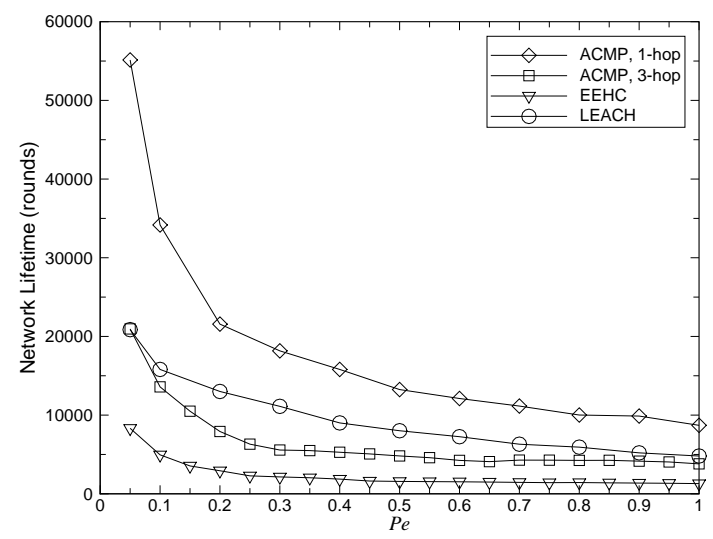

Fig. 13. The $P_{e}$ vs. network lifetime when $N=1000$.

This result encourages us to use ACMP especially in the area of frequent event appearance.

Fig. 13 shows that when the probability $P_{e}$ is low, the network lifetime of ACMP is long. This is because that, ACMP can distribute the traffic load in the network and balance the energy consumption well. However, the network lifetime of ACMP, EEHC, and LEACH are getting lower as $P_{e}$ increases higher. The ACMP 1-hop can obtain a longer network lifetime than EEHC's and LEACH's in higher network density due to local re-clustering scheme to prolong the network lifetime. From these results, we can conclude that a lower energy consumption can be obtained by decreasing the sensor density accordingly.

\section{CONCLUSION}

This paper presents an autonomous clustering and message passing (ACMP) protocol for energy efficiency in wireless sensor networks. The lifetime of the sensor network can be prolonged further by adopting an efficient traffic balance scheme. ACMP provides the load distribution scheme by maintaining the remaining energy to extend the network life time. Meanwhile, ACMP also provides a local re-clustering to avoid a node runs out of its energy when its remaining energy is low. Besides, ACMP uses the minimum energy consumption route (direct transmission) rather than uses multihop minimum distance route to form the cluster. Simulation results show that ACMP can achieve a highly energy saving effect as well as prolong the network lifetime.

\section{REFERENCES}

[1] A. D. Amis, R. Prakash, T. H. P. Vuong, and D. T. Huynh, "Max-Min D-Cluster Formation in Wireless Ad Hoc Networks," in Proc. IEEE INFOCOM 2000, vol. 1, pp. 32-41, Tel Avlv, Israel, Mar. 2000.

[2] S. Bandyopadhyay and E. J. Coyle, "Minimizing Communication Costs in Hierarchically Clustered Networks of Wireless Sensors," in Proc. IEEE WCNC 2003, vol. 2, pp. 1274-1279, New Orleans, LA, Mar. 2003.

[3] M. Bhardwaj, T. Garnett, and A. P. Chandrakasan, "Upper Bounds on the Lifetime of Sensor Networks," in Proc. IEEE ICC 2001, vol. 3, pp. 785-790, Helsinki, Finland, June 2001.

[4] B. Chen, K. Jamieson, H. Balakrishnan, and R. Morris, "Span: An Energy-Efficient Coordination Algorithm for Topology Maintenance in Ad Hoc Wireless Networks," ACM/Kluwer Wireless Networks, vol. 8, no. 5, pp. 481-492, Sept. 2002.

[5] J. Chen and L.-R. Li, "Path Planning Protocol for Collaborative Multirobot Systems," in Proc. IEEE CIRA'2005, Espoo, Finland, June 2005.

[6] Z. Chen and A. Khokhar, "Self Organization and Energy Efficient TDMA MAC Protocol by Wake Up For Wireless Sensor Networks," in Proc. IEEE SECON 2004, pp. 335-341, Santa Clara, CA, Oct. 2004.

[7] S. Bandyopadhyay and E. J. Coyle, "An Energy Efficient Hierarchical Clustering Algorithm for Wireless Sensor Networks," in Proc. IEEE INFOCOM 2003, vol. 3, pp. 1713-1723, San Francisco, CA, Mar./Apr. 2003.

[8] K. Dasgupta, K. Kalpakis, and P. Namjoshi, "An Efficient ClusteringBased Heuristic for Data Gathering and Aggregation in Sensor Networks," in Proc. IEEE WCNC 2003, vol. 3, pp. 1948-1953, New Orleans, LA, Mar. 2003.

[9] D. Estrin, R. Govindan, J. Heidemann, and S. Kumar, "Next Century Challenges: Scalable Coordination in Sensor Networks," in Proc. ACM/IEEE MobiCom'99, pp. 263-270, Seattle, WA, Aug. 1999.

[10] G. Gupta and M. Younis, "Load-Balanced Clustering of Wireless Sensor Networks," in Proc. IEEE ICC 2003, vol. 3, pp. 1848-1852, Anchorage, AK, May 2003.

[11] W. R. Heinzelman, A. Chandrakasan, and H. Balakrishnan, "EnergyEfficient Communication Protocol for Wireless Microsensor Networks," in Proc. Hawaii Int. Conf. Systems Sciences 2000, vol. 2, pp. 1-10, Maui, Hawaii, Jan. 2000.

[12] X. Ji, H. Zha, J. J. Metzner, and G. Kesidis, "Dynamic Cluster Structure for Object Detection and Tracking in Wireless Ad-Hoc Sensor Networks," in Proc. IEEE ICC 2004, vol. 7, pp. 3807-3811, Paris, France, June 2004.

[13] J. Kulik, W. Rabiner, and H. Balakrishnan, "Adaptive Protocols for Information Dissemination in Wireless Sensor Networks," in Proc. ACM/IEEE MobiCom'99, pp. 174-185, Seattle, WA, Aug. 1999.

[14] S. S. Kulkarni, "TDMA Service for Sensor Networks," in Proc. IEEE DCS Wksp. 2004, pp. 604-609, Tokyo, Japan, Mar. 2004.

[15] R. Min, et. al, "An Architecture For a Power-Aware Distributed Microsensor Node," in Proc. IEEE SiPS'00, pp. 581-590, Lafayette, LA, Oct. 2000.

[16] T. S. Rappaport, Wireless Communications: Principles and Practice, Prentice Hall PTR, Upper Saddle River, New Jersey, 1996.

[17] Y. E. Sagduyu and A. Ephremides, "The Problem of Medium Access Control in Wireless Sensor Networks," IEEE Wireless Commun., vol. 11, no. 6, pp. 44-53, Dec. 2004.

[18] A. Sinha and A. P. Chandrakasan, "Energy Aware Software," in Proc. Int. Conf. VLSI Design 2000, pp. 50-55, Calcutta, India, Jan. 2000.

[19] Y. Xu, J. Heidemann, and D. Estrin, "Geography-Informed Energy Conservation for Ad Hoc Routing," in Proc. ACM/IEEE MobiCom 2001, pp. 70-84, Rome, Italy, July 2001.

[20] W. Ye, J. Heidemann, and D. Estrin, "An Energy-Efficient MAC Protocol for Wireless Sensor Networks," in Proc. IEEE INFOCOM 2002, vol. 3, pp. 1567-1576, New York, USA, June 2002.

[21] O. Younis and S. Fahmy, "HEED: A Hybrid, Energy-Efficient, Distributed Clustering Approach for Ad Hoc Sensor Networks," IEEE Trans. Mobile Comput., vol. 3, no. 5, pp. 366-379, Oct./Dec. 2004. 